\title{
Time-domain investigation of an external cloak for antiplane elastic waves
}

\author{
S. Guenneau ${ }^{1}$, B. Lombard ${ }^{2}$, C. Bellis ${ }^{2}$ \\ 1 UMI 2004 Abraham de Moivre-CNRS, Imperial College London, SW7 2AZ, United Kingdom and \\ ${ }_{2}$ Aix Marseille Univ, CNRS, Centrale Marseille, LMA, Marseille, France
}

\begin{abstract}
Space folding techniques based on non-monotonic transforms lead to a new class of 2D isotropic cloaks with a constant negative shear modulus and a spatially varying negative density for antiplane elastic waves. We consider an external cloak consisting of a core with positive shear modulus and density, and a shell with simultaneously negative shear modulus and density. Such a core-shell resonant system creates a virtual folded region outside the shell. To handle such negative physical parameters in the time-domain, a two-step strategy is used: (i) assuming resonant (Drude and Lorentz-types) effective parameters in the frequency-domain; (ii) returning to the time-domain by applying the formalism of the auxiliary fields. We numerically show that, at the designed central frequency, scattering of a cylindrical antiplane elastic wave incident upon a finite set of small clamped obstacles is drastically reduced after a lapse of time, when they are placed in the close neighborhood of the external cloak. However, cloaking efficiency decreases when the source is placed in the intense near field of the external cloak and moreover at short times, cloaking breaks down.
\end{abstract}

It is well established that one can cloak objects surrounded by specially designed anisotropic heterogeneous [1-5], or plasmonic [6-8], shells. It is even possible to cloak objects using some active sources $[9,10]$. In this Letter, we analyze the possibility to reduce the scattering of small objects placed outside a core-shell system with sign-shifting heterogeneous (yet isotropic) coefficients.

Here, we focus on the case of 2D scalar waves propagating in non-dissipative media governed by equations:

$$
\left\{\begin{array}{l}
\partial_{t} \boldsymbol{\sigma}(\boldsymbol{x}, t)=\mu(\boldsymbol{x}) \boldsymbol{\nabla} v(\boldsymbol{x}, t), \\
\rho(\boldsymbol{x}) \partial_{t} v(\boldsymbol{x}, t)=\boldsymbol{\nabla} \cdot \boldsymbol{\sigma}(\boldsymbol{x}, t) .
\end{array}\right.
$$

Such a system has a wide range of applicability [11], such as in antiplane elasticity where $v$ is the out-ofplane velocity $\left(\mathrm{m} \cdot \mathrm{s}^{-1}\right), \boldsymbol{\sigma}$ the stress $(\mathrm{Pa}), \rho$ the mass density $\left(\mathrm{kg} \cdot \mathrm{m}^{-3}\right)$ and $\mu$ the elasticity modulus $(\mathrm{Pa})$ of the medium, all of which depend upon the space and time variables $\boldsymbol{x}=\left(x_{1}, x_{2}\right) \in \mathbb{R}^{2}$ and $t \geq 0$, with $\boldsymbol{\nabla}=\left(\partial / \partial x_{1}, \partial / \partial x_{2}\right)^{T}$.

Inspired by earlier work on anomalous resonance and cloaking with resonant core-shell systems based on the electrostatic [12-18] and electromagnetic [19-21] settings of equation (1) in the time-harmonic regime, we consider the following set of radially symmetric parameters for an external cloak for the 2D scalar wave system (1) with core and shell radii $R_{c}<R_{s}$ (see Fig. 2):

$$
\begin{gathered}
\mu^{-1}\left(\boldsymbol{x} ; \omega_{0}\right)=\mu_{e}^{-1} \begin{cases}+1 & 0 \leq|\boldsymbol{x}|<R_{c}, \\
-1 & R_{c}<|\boldsymbol{x}| \leq R_{s}, \\
+1 & R_{s}<|\boldsymbol{x}|,\end{cases} \\
\rho\left(\boldsymbol{x} ; \omega_{0}\right)=\rho_{e} \begin{cases}+\left(R_{s} / R_{c}\right)^{4} & 0 \leq|\boldsymbol{x}|<R_{c}, \\
-\left(R_{s} /|\boldsymbol{x}|\right)^{4} & R_{c}<|\boldsymbol{x}| \leq R_{s}, \\
+1 & R_{s}<|\boldsymbol{x}|,\end{cases}
\end{gathered}
$$

with $|\boldsymbol{x}|=\sqrt{x_{1}^{2}+x_{2}^{2}}$ and $\mu_{e}, \rho_{e}>0$, while the central circular frequency parameter $\omega_{0}$ emphasizes that (2) per- tains to the wave equation in the frequency-domain. We note these material parameters can be inferred from the analogous transverse electromagnetic problem [20, 21] where the electric permittivity plays the role of $\mu^{-1}$ (resp. $\rho$ ) and the magnetic permeability that of $\rho\left(\right.$ resp. $\left.\mu^{-1}\right)$ in $s$ (resp. $p$ ) light polarization in (1). Although our elastic parameters are concerned with antiplane elastic waves in transformed 2D elastic media [24, 25], results of our study can thus be translated to transverse electromagnetic waves. The specific form of (2) achieves impedancematching at the core and shell interfaces and has been related in $[15,20,21]$ (albeit in an electrostatic/timeharmonic electromagnetic configuration) to coordinate transform $\phi: \boldsymbol{x} \mapsto \boldsymbol{x}^{\prime}$ defined as:

$$
\left|\boldsymbol{x}^{\prime}\right|=\phi(|\boldsymbol{x}|)= \begin{cases}R_{s}^{2}|\boldsymbol{x}| / R_{c}^{2} & \text { for }|\boldsymbol{x}| \leq R_{c}, \\ R_{s}^{2} /|\boldsymbol{x}| & \text { for } R_{c}<|\boldsymbol{x}| \leq R_{s}, \\ |\boldsymbol{x}| & \text { for }|\boldsymbol{x}|>R_{s} .\end{cases}
$$

Unlike in usual transformation acoustics where mass density is mapped, in general, to a rank-2 tensor while bulk modulus remains a scalar [23], we note that the use of coordinate transform (3) leads to transformed constitutive parameters $\mu^{\prime}\left(\boldsymbol{x}^{\prime} ; \omega_{0}\right)$ and $\rho^{\prime}\left(\boldsymbol{x}^{\prime} ; \omega_{0}\right)$ that remain scalar functions in each subdomain. Geometrically, $\phi$ maps the core $\operatorname{disc} \mathcal{C}=D\left(0, R_{c}\right)$ onto $D\left(0, R_{*}\right)$, with $R_{*}=R_{s}^{2} / R_{c}$, and folds the shell annulus $\mathcal{S}=D\left(0, R_{s}\right)-D\left(0, R_{c}\right)$ onto $D\left(0, R_{s}\right)-D\left(0, R_{*}\right)$, see Fig. 2 . Note that $\phi$ in $(3)$ is a non-bijective (and non-smooth) function, which is a genuine feature of space folding.

Thanks to $\phi$ in (3), we thus create a folding zone in space, namely the annulus $\mathcal{F}_{*}$ of width $\left|R_{s}-R_{*}\right|$, which corresponds to the so-called external cloaking region $\mathcal{F}$ in the physical space [20] (with $\mathcal{F}_{*} \equiv \mathcal{F}$ ). Physically, this means that the scalar wavefield outside the region of radius $R_{*}$ should not sense what is inside this region, and this has been confirmed by a mathematical analysis based on the wave equation in the time-harmonic regime [21]. Interestingly, the core-shell system (3) can also be viewed 


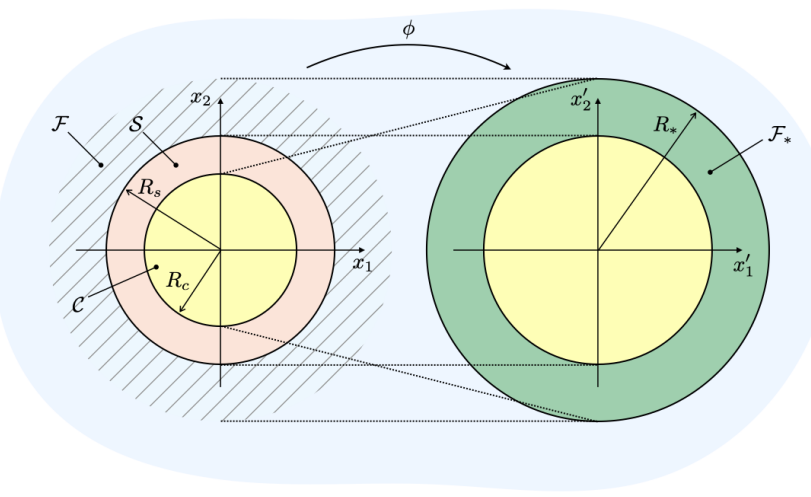

Figure 1: Left: schematics of core-shell system in physical domain with external cloaking region $\mathcal{F}$ (dashed area). Right: transformed domain with virtual folded region $\mathcal{F}_{*}$ (in green).

as a folded version of the Veselago-Pendry lens [26-28], via the concept of complementary media [29], which is reminiscent of space-folding. Actually, it has been numerically shown in the time-harmonic regime that if one considers a finite size object outside the external cloak and a "space folded" copy of the object consisting of opposite sign electromagnetic parameters inside the shell, then this makes a perfectly transparent electromagnetic core-shell system at any frequency $[30,31]$.

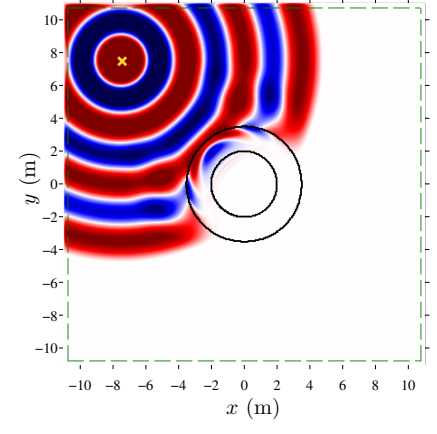

(a) $t=35.6 \mathrm{~ms}$.

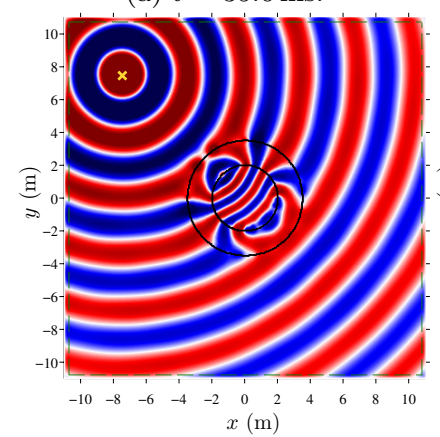

(c) $t=1.424 \mathrm{~s}$.

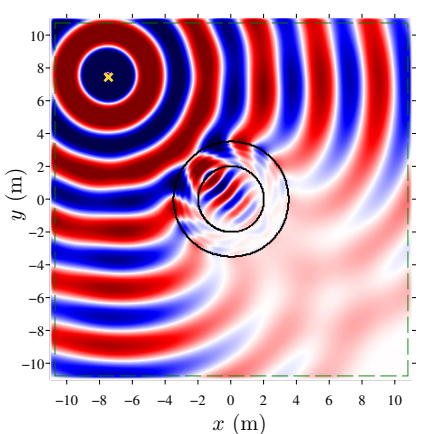

(b) $t=71.2 \mathrm{~ms}$

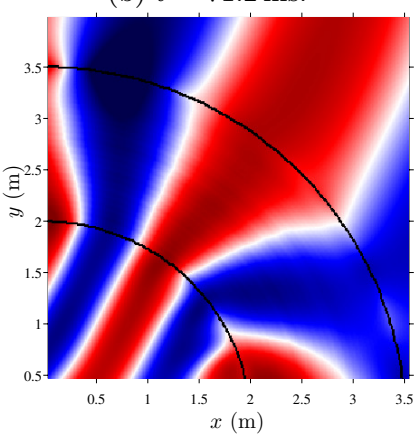

(d) $t=1.424 \mathrm{~s}$ close-up.
Figure 2: Snapshots of wavefield $v$ at different times for the cloak only (multimedia view in Cloak-final.avi). The yellow cross marks the source. Panel (d) is a close-up of (c) highlighting negatively refracted waves in the isotropic shell.

However, despite all these works, the transient behavior of waves propagating across the core-shell system (2) remained an open question until now, and we would like to address it through time-domain numerical simulations. To do so, we employ the formalism of auxiliary fields, which has proved powerful for modeling electromagnetic [32-34] and acoustic [35] waves propagating within signshifting media.

In the core $\mathcal{C}$ and in the matrix $\mathbb{R}^{2} \backslash(\mathcal{C} \cup \mathcal{S})$, the physical parameters $\mu\left(\boldsymbol{x} ; \omega_{0}\right)$ and $\rho\left(\boldsymbol{x} ; \omega_{0}\right)$ in $(2)$ are both positive, which meets the standard assumption for a mathematically, and physically, well-posed problem. On the other hand, in the shell $\mathcal{S}$, both $\mu\left(\boldsymbol{x} ; \omega_{0}\right)$ and $\rho\left(\boldsymbol{x} ; \omega_{0}\right)$ are negative, which is non-standard and requires special treatment in order to investigate external cloaking in the time-domain. To do so, we consider that such negative parameters can be realized, at the given frequency $\omega_{0}$, as the macroscopic (homogenized) behavior in the timeharmonic regime of a resonant microstructured medium (which we do not intend to describe here) filling this region of space. Resonant microstructures generally lead to frequency-dependent effective constitutive parameters:

$$
\kappa(\boldsymbol{x}, \omega)=\kappa\left(\boldsymbol{x} ; \omega_{0}\right) g_{\kappa}(\omega),
$$

for $\kappa=\mu^{-1}, \rho$ and $\boldsymbol{x} \in \mathcal{S}$. The introduction of $g_{\kappa}$ allows for the possible use of two different resonant mechanisms on $\mu$ and $\rho$, with the constraint that $g_{\kappa}\left(\omega_{0}\right)=-1$, as they usually rely upon different structural elements inside acoustic metamaterials, see e.g. [36, 37] for passive structures and [38] for active circuits. Considering first a single Drude-type model, we set $g_{\mu^{-1}}=g_{\rho}=1-\Omega_{0}^{2} / \omega^{2}$ with $\Omega_{0}=\sqrt{2} \omega_{0}$. We note that even if such a Drude model presents some shortcomings for effective elastic parameters, it is an accurate plasmonic model for the electric permittivity [39]. To handle such frequency-dependent parameters in the time-domain wave equation, we use the formalism of auxiliary fields [35]. By introducing locally the fields $\boldsymbol{\psi}$ and $w$, elastic equations in the shell $\mathcal{S}$ are rewritten as:

$$
\left\{\begin{array}{l}
\mu_{e}^{-1} \partial_{t} \boldsymbol{\sigma}+\mu_{e}^{-1} \Omega_{0}^{2} \partial_{t} \boldsymbol{\psi}+\boldsymbol{\nabla} v=\mathbf{0} \\
\rho\left(\boldsymbol{x} ; \omega_{0}\right) \partial_{t} v+\rho\left(\boldsymbol{x} ; \omega_{0}\right) \Omega_{0}^{2} \partial_{t} w+\boldsymbol{\nabla} \cdot \boldsymbol{\sigma}=0 \\
\partial_{t t}^{2} \boldsymbol{\psi}=\boldsymbol{\sigma} \\
\partial_{t t}^{2} w=v
\end{array}\right.
$$

where we emphasize only the variable dependency of the constitutive parameters. Equations (5) are completed with (1) in the core $\mathcal{C}$ and in the matrix $\mathbb{R}^{2} \backslash(\mathcal{C} \cup \mathcal{S})$ with the parameters in (2). We further assume the usual continuity conditions of $v$ and $\boldsymbol{\sigma} \cdot \boldsymbol{n}$ at the core and shell interfaces, with $\boldsymbol{n}$ the unit normal vector at the interfaces, and suitable outgoing wave conditions at infinity.

Some scatterers are positioned in the cloaking region $\mathcal{F}$. These are nine equidistant and identical circular obstacles of radius $R$ whose centre is at a distance $d$ from the centre of the cloak. In the tracks of [13-21], we consider small scatterers, and thus a homogeneous Dirichlet 


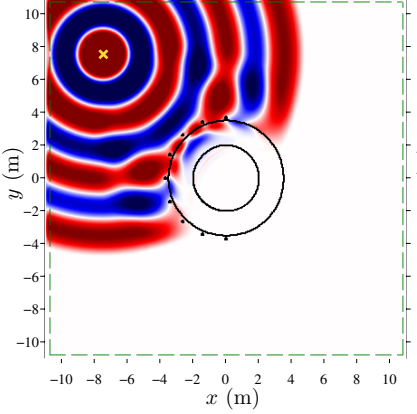

(a) $t=35.6 \mathrm{~ms}$.

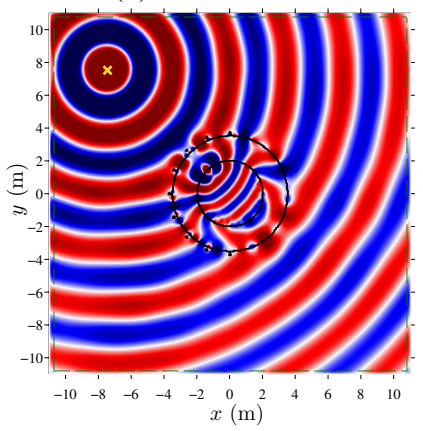

(c) $t=1.424 \mathrm{~s}$.

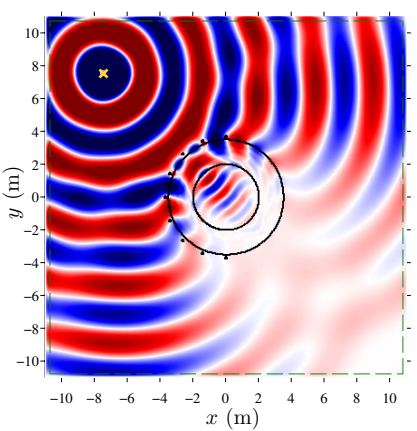

(b) $t=71.2 \mathrm{~ms}$.

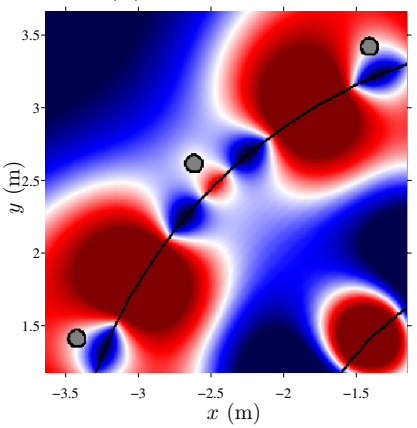

(d) $t=1.424 \mathrm{~s}$ close-up.
Figure 3: Snapshots of wavefield $v$ at different times for scatterers with the cloak (multimedia view in Scatcloak-final.avi). The yellow cross marks the source. Panel (d) is a close-up of (c) highlighting the interactions of the surface waves on the shell with the scatterers placed in $\mathcal{F}$ (grey dots).

condition $v=0$ (typical of rigid inclusions in elasticity) is imposed on the obstacles to maximize the scattered wavefield and thus emphasize the effects of the cloak. Indeed, the amplitude of the wavefield scattered by small holes (with Neumann boundary condition $\boldsymbol{\sigma} \cdot \boldsymbol{n}=0$ ) is comparatively much smaller [40], which reduces de facto the efficiency of the cloak (see supplementary material).

Parameters are chosen as $R_{c}=2 \mathrm{~m}, R_{s}=3.5 \mathrm{~m}, d=$ $3.7 \mathrm{~m}, R=0.0583 \mathrm{~m}, \mu_{e}=1.2 \cdot 10^{8} \mathrm{~Pa}, \rho_{e}=10^{3} \mathrm{~kg} \cdot \mathrm{m}^{-3}$. The cloaking region $\mathcal{F}$ is an annulus of width $\left|R_{s}-R_{*}\right|=$ $\left|R_{s}-R_{s}^{2} / R_{c}\right|=2.625 \mathrm{~m}$. The scatterers are put with a uniform polar angle $\theta$, from $\theta=\pi / 2$ to $3 \pi / 2 \mathrm{rad}$, and are illuminated by a source at point $\boldsymbol{x}_{s}=(-7.5,7.5) \mathrm{m}$ that emits a monochromatic signal at the central frequency $f_{0}=\omega_{0} / 2 \pi=100 \mathrm{~Hz}$, switched on at time $t=0 \mathrm{~s}$.

Systems (1) and (5) are solved on a Cartesian computational grid with $N_{1} \times N_{2}$ points. The grid is deemed fine enough with $N_{1}=N_{2}=1700$ to ensure a sufficient number of points discretizing the scatterers. A 4th order space-time ADER scheme is used with a splitting algorithm. Discretization of curved interfaces on the Cartesian grid considered requires care to avoid spurious diffraction and to accurately account for jump conditions. To do so, the Explicit Simplified Interface Method is used [41]. This method is based on the coupling at each time step between two meshes: a Cartesian 2D mesh where equations (5) are discretized, and a curved $1 \mathrm{D}$ mesh of the interface. The coupling is achieved by imposing strong jump conditions and by using the local geometry of the interface. Most of the work is done in pre-processing, before numerical integration, leading to a low computational cost. Technical details are provided in [42]. Perfectly-matched layers are applied on external boundaries of computational domain to simulate outgoing waves. Computations are performed using PROSPERO (http://prospero-software.science/). All wave patterns shown in the sequel use a common normalization and the same linear color scale (ranging from dark blue for minimal values of field to dark red for its maximum values).

In our first numerical experiment, we consider the cloak only, i.e. without scatterers. Figure 2 illustrates the interaction of the transient waves with the cloak at different time steps. After a transient period (a-b), cloaking takes place (c). In (d), a close-up shows waves inside the shell. The effect of space folding can be observed. Dynamic animations (supplementary material) show the propagation of negatively refracted waves within the shell $\mathcal{S}$, where phase and group velocity have opposite signs since the constitutive parameters $\mu$ and $\rho$ are simultaneously negative (albeit being spatially varying) according to (2). However, within the core $\mathcal{C}, \mu$ and $\rho$ are both positive constant, but the refractive index therein is larger than in the medium surrounding the cloak, which leads to an enhanced power flow in $\mathcal{C}$, similar to that in concentrators [43]. Finally, anomalous resonances at the core-shell $\left(|\boldsymbol{x}|=R_{c}\right)$ and shell-outer medium $\left(|\boldsymbol{x}|=R_{s}\right)$ interfaces of such a core-shell system predicted 25 years ago for an electrostatic configuration in [12] manifest here a wave character since the condition for existence of surface waves akin to those already studied in the context of impedance-matched and sign-shifting electromagnetic media $[29,44,45]$, is naturally satisfied at all times by the principle of space-folding.

Figure 3 illustrates the interaction of transient waves with the scatterers and the cloak, at different time steps. Clearly, at short times, the core-shell system does not cloak the scatterers, and behaves in a way similar to a superscatterer [46], whereas at longer times, when some permanent regime has been established, the diffraction by the scatterers is much reduced. A close-up on the scatterers in panel (d) shows the interaction of surface waves, at the matrix-shell interface, with the scatterers in the cloaking region $\mathcal{F}$ (see supplementary material). We note in $(d)$ that scatterers are at the nodal lines of surface waves, and thus scatterers have minimal scattering.

Figure 4 displays snapshots of the scattered wavefield $v$ at $t=1.424 \mathrm{~s}$ in various configurations. The diffraction patterns of the scatterers (a) are significantly reduced by the cloak (b). In (c), the distance between the cloak and the scatterers is increased to $d=4.7 \mathrm{~m}$ : the interaction between surface waves and the scatterers is weaker, which degrades the efficiency of the cloak. This degradation is amplified when larger scatterers are considered, as in (d) 


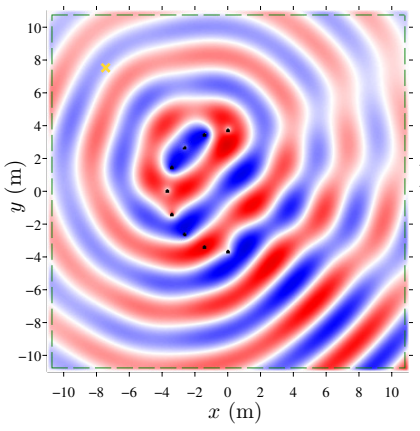

(a) Scatterers $(R=0.0583$ $\mathrm{m})$ at $d=3.7 \mathrm{~m}$.

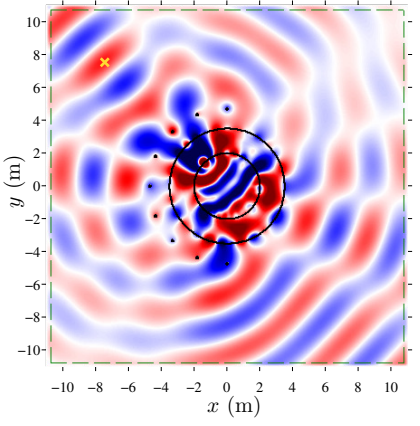

(c) Same as (b), with $d=4.7 \mathrm{~m}$.

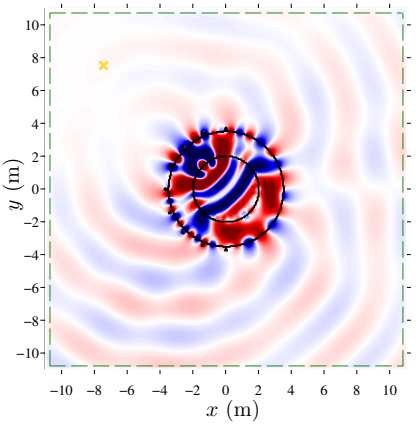

(b) Same as (a) with cloak.

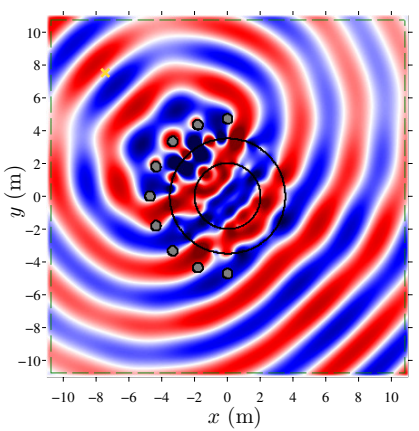

(d) Same as (c), with $R=0.3 \mathrm{~m}$.
Figure 4: Snapshots of scattered wavefield $v$ at time $t=$ $1.424 \mathrm{~s}$ with the source (yellow cross) at $\left|\boldsymbol{x}_{s}\right|=10.6 \mathrm{~m}$.

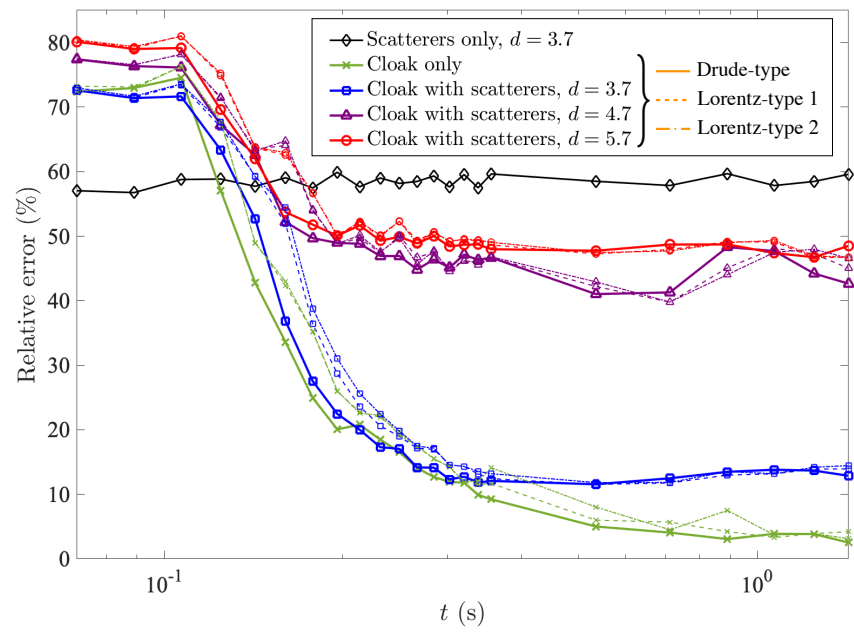

Figure 5: $L^{2}$-norm of the scattered wavefield $v$ in $\Omega_{\text {obs }}$ as a function of time (in log scale) for various configurations. The scatterers radius is $R=0.0583 \mathrm{~m}$ and their distance to the cloak center is $d$ (in meters).

with $R=0.3 \mathrm{~m}$. These act somehow as a screen that prevents waves from reaching the cloak, so that surface waves cannot be generated to reduce scattering. One might argue that a possible route to achieve invisibility for larger scatterers, or even finite size possibly heterogeneous objects, that might be located further away from the cloak, would be to implement an external cloak based on complementary media as in [30]. But this would imply that the design of the external cloak depends upon the objects to cloak, unlike in the present scheme. We further note that one can in theory increase ad libitum the radius $R_{*}$ of the cloaking region by considering a vanishing radius $R_{c}$ for the core, which according to (2) requires a core where $\rho \rightarrow \infty$. This might open a route to external cloaking of finite size objects, or objects located further away from the cloak.

To quantify these observations, the instantaneous $L^{2}$ norm $\|\cdot\|=\left(\int_{\Omega_{\mathrm{obs}}}|\cdot|^{2} d \boldsymbol{x}\right)^{\frac{1}{2}}$ of the fields is measured in the rectangular domain $\Omega_{\mathrm{obs}}=[-10,10] \times[-10,-7] \mathrm{m}^{2}$ opposite the source point. From now on, the relative error is defined as the ratio $\left\|v-v_{\text {inc }}\right\| /\left\|v_{\text {inc }}\right\|$, with $v$ the solution to (1) and (5) and $v_{\text {inc }}$ being the associated incident wavefield in the background medium. This relative error is reported in Fig. 5 and plotted as a function of time and for some of the various configurations considered previously. Without the cloak, a steady state is reached rapidly and leads to a relative error around $60 \%$. With cloak and scatterers at $d=3.7 \mathrm{~m}$, steady state is reached only at about $0.3 \mathrm{~s}$, i.e. 30 periods of the source. Once the steady state is reached, external cloaking is all the more efficient than scatterers are close to core-shell system, as discussed previously: at $d=3.7 \mathrm{~m}$, relative error is around $12 \%$. For comparison, the cloak alone yields an error around $2.5 \%$. In addition to the error measurements corresponding to the chosen single Drude-type model (solid lines), we also report in Fig. 5 some additional results (dashed lines) corresponding to the choice of two different Lorentz-type models in (4):

$$
g_{\kappa}(\omega)=1-\Omega_{\kappa, 0}^{2} /\left(\omega^{2}-\Omega_{\kappa, r}^{2}\right)
$$

with resonances at non-zero frequencies $\Omega_{\kappa, r}$, while we set $\Omega_{\kappa, 0}=\left(2\left(\omega_{0}^{2}-\Omega_{\kappa, r}^{2}\right)\right)^{\frac{1}{2}}$ so that $g_{\kappa}\left(\omega_{0}\right)=-1$. The parameters are chosen as $\Omega_{\mu^{-1}, r}=100 \pi, \Omega_{\rho, r}=40 \pi$ rad.s ${ }^{-1}$, for Lorentz-type 1 and $\Omega_{\mu^{-1}, r}=40 \pi, \Omega_{\rho, r}=$ $100 \pi$ rad.s ${ }^{-1}$, for Lorentz-type 2 . System (5) is modified accordingly. Associated results in Fig. 5 show the same trends as the single Drude-type model, highlighting the low sensitivity of the overall behavior of the cloak to the specific choice of the resonant mechanisms in the shell. We finally note the long time behaviour would depend on the lapse of time the source has been on as well as inherent losses in the negatively refracting shell $[47,48]$.

Considering the configuration with $R=0.0583 \mathrm{~m}$ and $d=3.7 \mathrm{~m}$, we now vary the distance $\left|\boldsymbol{x}_{s}\right|$ of the source to the center of the cloak (with the single Drude-type model), from $\left|\boldsymbol{x}_{s}\right|=4 \mathrm{~m}$, when the source lies in the intense near field of the shell (cf. Fig. 6a-c) to $\left|\boldsymbol{x}_{s}\right|=6.2 \mathrm{~m}$, when it lies just outside the cloaking annulus of outer radius $R_{*}=6.125 \mathrm{~m}$ (see also Fig. $6 \mathrm{~d}$ for an intermediate case). The corresponding relative errors are reported in Figure 7a. For $\left|\boldsymbol{x}_{s}\right| \geq 5 \mathrm{~m}$ the cloaking performances of the core-shell system appear to be comparable to the case where the source is much further away (see Fig. 4b and 5 where $\left|\boldsymbol{x}_{s}\right|=10.6 \mathrm{~m}$ ). These performances deteriorate 


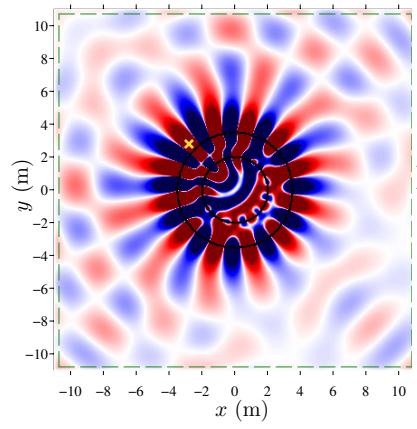

(a) $\left|\boldsymbol{x}_{s}\right|=4 \mathrm{~m}$, cloak alone.

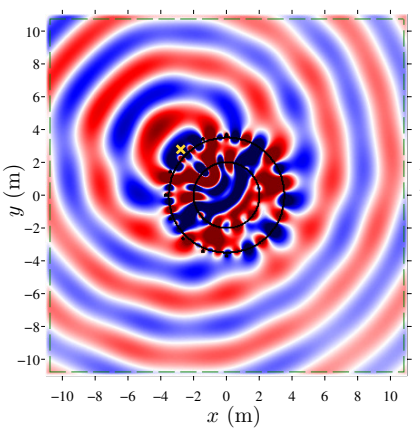

(c) Same as (b) with cloak.

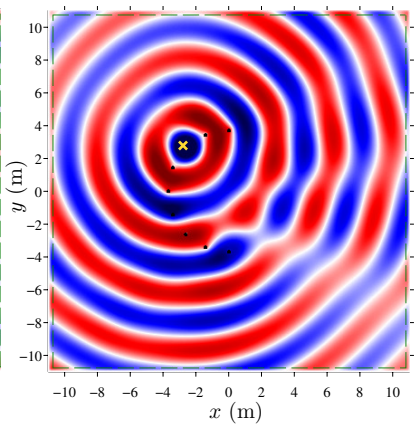

(b) $\left|\boldsymbol{x}_{s}\right|=4 \mathrm{~m}$, scatterers $(R=0.0583 \mathrm{~m}$ at $d=3.7 \mathrm{~m})$

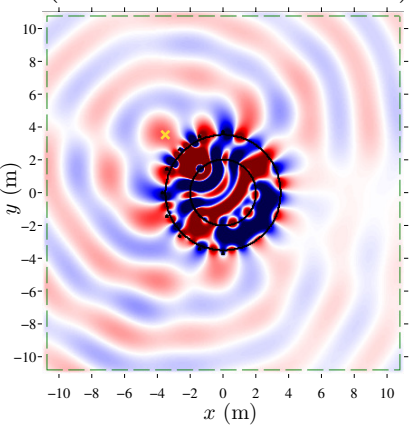

(d) Same as (c) for $\left|\boldsymbol{x}_{s}\right|=5 \mathrm{~m}$.
Figure 6: Snapshots of scattered wavefield $v$ at time $t=$ $1.424 \mathrm{~s}$ depending on the source distance $\left|\boldsymbol{x}_{s}\right|$ to the cloak center. The yellow cross marks the source.

when the source approaches the shell, with a sharp increase in the error when the former enters the region over which the surface waves extend. Estimating such a skin depth is a delicate open question.

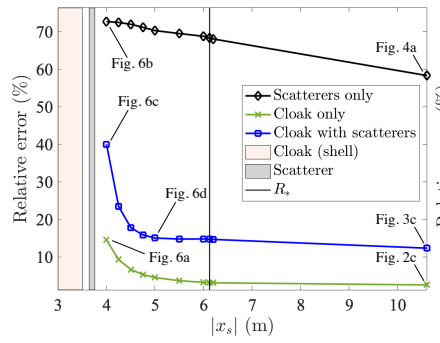

(a) Relative error (\%) vs source distance to the cloak.

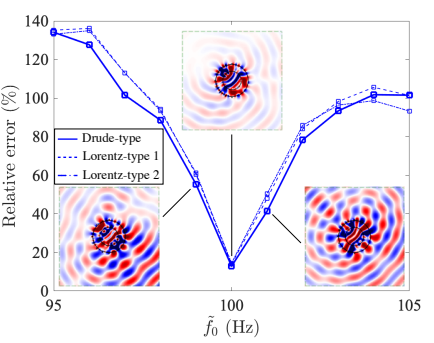

(b) Relative error (\%) vs source central frequency and some scattered fields.
Figure 7: Instantaneous $L^{2}$-norm at $t=1.424 \mathrm{~s}$ of scattered wavefield in $\Omega_{\text {obs }}$ as a function of some source parameters.

To achieve desired negative physical parameters in the shell, we emulate them first as a realization of a single effective Drude medium. The coefficient $\Omega_{0}$ in (4) was defined from frequency $f_{0}=100 \mathrm{~Hz}$. Figure $7 \mathrm{~b}$ then represents relative errors measured when the central frequency of the source is shifted as $\tilde{f}_{0}$ (solid line). Cloaking performances are significantly reduced when the incident frequency is not tuned so that properties (2) are met within the shell, which highlights sensitivity of cloaking to physical parameters used: injecting $f=95 \mathrm{~Hz}$ in (4) yields $g\left(0.95 \omega_{0}\right)=-1.21$, which brings a deviation of only $21 \%$ from (2) but in turn a sharp increase of the error to $134 \%$. Repeating this analysis with the two different Lorentz-type models (6) (dashed lines) yields the same trends, confirming earlier observations. We note that a similar breakdown of external cloaking away from the source central frequency has been observed in [50], see also [49] for similar issues with superlensing.

These efficiency measurements as a function of frequency raise three questions: (i) How to emulate negative parameters in the time-domain over a large frequency bandwidth? (ii) How to realize such a cloak in practice using a resonant microstructured medium within the shell? An interesting route towards fabrication of such complementary media has been proposed in [36-38], albeit only tested in the frequency domain. (iii) Would a metamaterial external cloak work for short-time elastic and electromagnetic pulses since this might open interesting applications in imaging, sensing, and detection? These questions, crucial for the design of external cloaks in the time-domain, are left for future investigations.

Supplementary Material: The supplementary material contains additional numerical data (Figures and Tables) that support the findings in this study. For instance, we consider scatterers with Neumann datum on their boundary and we also implement constitutive parameters following a Lorentz-type law. Movie files are also available as accompanying Multimedia for Figures 2 and 3.

We acknowledge the help of Dr. Patrick Sanchez from LMA to generate movie files (supplementary material).

[1] J.B. Pendry, D. Schurig and D.R. Smith, Controlling electromagnetic fields, Science 312, 1780-1782 (2006).

[2] U. Leonhardt and T.G. Philbin 2006. General relativity in electrical engineering. New J. Phys. 8, 247 (2006).

[3] G.W. Milton, M Briane, JR Willis, On cloaking for elasticity and physical equations with a transformation invariant form, New J. Phys. 8, 248 (2006).

[4] A. Greenleaf, M. Lassas, and G. Uhlmann. Anisotropic conductivities that cannot be detected by EIT. Physiol. Meas. 24, 413-419 (2003).

[5] H. Chen and C.T. Chan, Acoustic cloaking and transformation acoustics, J. Phys. D: Appl. Phys. 43113001 (2010).

[6] A. Alù, and N. Engheta, Achieving transparency with plasmonic and metamaterial coatings, Phys. Rev. E 72, 016623 (2005).

[7] P.Y. Chen, M. Farhat, S. Guenneau, S. Enoch and A. Alù, Acoustic scattering cancellation via ultrathin pseudo-surface, App. Phys. Lett. 99, 191913 (2011).

[8] X. Zhu, B. Liang, W. Kan, X. Zou and J. Cheng, Acoustic Cloaking by a Superlens with Single-Negative Materials, Phys. Rev. Lett. 106, 014301 (2011).

[9] D.A.B. Miller, On perfect cloaking, Opt. Express 14, 12457 (2006).

[10] F. Guevara Vasquez, G.W. Milton, D. Onofrei, Broadband exterior cloaking, Opt. Express 17, 14800 (2009).

[11] D. Joyce, Duncan, W. Parnell, R. Assier, and D. Abrahams, An integral equation method for the homogeniza- 
tion of unidirectional fibre-reinforced media; antiplane elasticity and other potential problems, Proc. Roy. Soc. A, 473, 2201, 20170080 (2017).

[12] N.A. Nicorovici, R.C. McPhedran, and G.W. Milton, Optical and dielectric properties of partially resonant composites, Phys. Rev. B 49, 8479 (1994).

[13] G.W. Milton and N.A. Nicorovici, On the cloaking effects associated with anomalous localized resonance, Proc. R. Soc. A 462, 3027-3059 (2006).

[14] O.P. Bruno and S. Lintner, Superlens-cloaking of small dielectric bodies in the quasistatic regime, J. Appl. Phys. 102, 124502 (2007).

[15] G.W. Milton and N. A. Nicorovici, R. C. McPhedran, K. Cherednichenko, and Z. Jacob, Solutions in folded geometries, and associated cloaking due to anomalous resonance, New J. Phys. 10, 115021 (2008).

[16] G. Bouchitté and B. Schweizer, Cloaking of small objects by anomalous localized resonance, Quart. J. Mech. Appl. Math. 63, 437-463 (2010)

[17] H. Ammari, G. Ciraolo, H. Kang, H. Lee and G.W. Milton, Spectral theory of a Neumann-Poincare-type operator and analysis of cloaking due to anomalous localized resonance. Arch. Rat. Mech. Anal. 208, 667-692 (2013).

[18] R. V. Kohn, J. Lu, B. Schweizer, and M.I. Weinstein, A variational perspective on cloaking by anomalous localized resonance, Comm. Math. Phys. 328, 1-27 (2014).

[19] N. A. Nicorovici, R.C. McPhedran, S. Enoch, and G. Tayeb, Finite wavelength cloaking by plasmonic resonance, New J. Phys. 10, 115020 (2008).

[20] Y. Liu, B. Gralak, R.C. McPhedran, and S. Guenneau, Finite frequency external cloaking with complementary bianisotropic media, Opt. Express 22, 1738717402 (2014).

[21] H.M. Nguyen, Cloaking an arbitrary object via anomalous localized resonance: the cloak is independent of the object. SIAM J. Math. Anal. 49, 3208-3232 (2017).

[22] S.A. Cummer and D. Schurig, One path to acoustic cloaking, New J. Phys. 9, 45 (2008).

[23] A.N. Norris, Acoustic cloaking theory, Proc. Roy. Soc. A 464, 2411-2434 (2008).

[24] W.J. Parnell, A.N. Norris and T. Shearer, Employing pre-stress to generate finite cloaks for antiplane elastic waves App. Phys. Lett. 100, 171907 (2012).

[25] D.J. Colquitt, I.S. Jones, N.V. Movchan, A.B. Movchan, M. Brun and R.C. McPhedran, Making waves round a structured cloak: Lattices, negative refraction and fringes, Proc R Soc A 469, 20130218 (2013).

[26] V.G. Veselago, The electrodynamics of substances with simultaneously negative values of $\varepsilon$ and $\mu$, Sov. Phys. Usp. 10 (4), 509-514 (1967).

[27] J.B. Pendry, Negative refraction makes a perfect lens. Phys. Rev. Lett. 85, 3966-3969 (2000).

[28] G.W. Milton, N.A. Nicorovici, R.C. McPhedran, and V.A. Podolskiy, A proof of superlensing in the quasistatic regime, and limitations of superlenses in this regime due to anomalous localized resonance, Proc. Roy. Soc. A 461, 3999-4034 (2005).

[29] J.B. Pendry and S. Anantha Ramakrishna, Near field lenses in two dimensions, J. Phys.: Condens. Matter 14, 8463-8479 (2002).

[30] Y. Lai, H. Chen, Z.-Q. Zhang and C.T. Chan, Complementary media invisibility cloak that cloaks objects at a distance outside the cloaking shell, Phys. Rev. Lett. 102, 093901 (2009)
[31] H.-M. Nguyen and L.H. Nguyen, Cloaking using complementary media for the Helmholtz equation and a three spheres inequality for second order elliptic equations, Trans. Amer. Math. Soc. B2 93-112 (2015).

[32] J.L. Young and R.0. Nelson, A Summary and Systematic Analysis of FDTD Algorithms for Linearly Dispersive Media, IEEE Antennas Propag. Mag. 43, 61-77 (2001).

[33] A. Raman and S. Fan, Photonic Band Structure of Dispersive Metamaterials Formulated as a Hermitian Eigenvalue Problem, Phys. Rev. Lett. 104, 087401 (2010).

[34] B. Gralak and A. Tip, Macroscopic Maxwell's equations and negative index materials, Journal of mathematical physics 51 (5), 052902 (2010).

[35] C. Bellis and B. Lombard, Simulating transient wave phenomena in acoustic metamaterials using auxiliary fields, Wave Motion 86, 175-194 (2019).

[36] C. Shen, J. Xu, N.X. Fang and Y. Jing, Anisotropic Complementary Acoustic Metamaterial for Canceling out Aberrating Layers, Phys. Rev. X 4, 041033 (2014)

[37] J. Wang, F. Allein, N. Boechler, J. Friend and O. Vazquez-Mena, Design and Fabrication of NegativeRefractive-Index Metamaterial Unit Cells for NearMegahertz Enhanced Acoustic Transmission in Biomedical Ultrasound Applications, Phys. Rev. Applied 15, 024025 (2021)

[38] S.R. Craig, P.J. Welch and C. Shi, Non-Hermitian complementary acoustic metamaterials for lossy barriers, Appl. Phys. Lett. 115, 051903 (2019).

[39] S.A. Maier, Plasmonics: fundamentals and applications, Springer, New-York (2007).

[40] P. Martin, Multiple Scattering: Interaction of TimeHarmonic Waves with N Obstacles, Cambridge University Press (2006).

[41] G. Chiavassa, B. Lombard, Time domain numerical modeling of wave propagation in $2 \mathrm{D}$ heterogeneous porous media, J. Comput. Phys. 230, 5288-5309 (2011).

[42] B. Lombard and J. Piraux, Numerical treatment of twodimensional interfaces for acoustic and elastic waves, Journal of Computational Physics, 195, 90-116 (2004).

[43] M. Rahm, D. Schurig, D.A. Roberts, S.A. Cummer, D.R. Smith and J.B. Pendry, Design of electromagnetic cloaks and concentrators using form-invariant coordinate transformations of Maxwell's equations, Photonics and Nanostructures: Fund. Appl. 6 (1), 87-95 (2008).

[44] S. Guenneau, B. Gralak and J.B. Pendry, Perfect corner reflector, Opt. letters 30 (10), 1204-1206 (2005).

[45] A.-S. Bonnet-Ben Dhia, P. Ciarlet Jr., and C.M. Zwolf, Time harmonic wave diffraction problems in materials with sign-shifting coefficients, J. Comput. Appl. Math., 234, 1912-1919 (2010).

[46] T. Yang, H. Chen, X. Luo, and H. Ma, Superscatterer: Enhancement of scattering with complementary media, Opt. Express 16, 18545 (2008).

[47] A.D. Yaghjian and T.B. Hansen, Plane-wave solutions to frequency-domain and time-domain scattering from magnetodielectric slabs, Phys. Rev. E 73, 046608 (2006).

[48] G.W. Milton, N.-A. P. Nicorovici, R.C. McPhedran, Opaque perfect lenses, Physica B 394, 171-175 (2007).

[49] A. Archambault, M. Besbes, and J.-J. Greffet, Superlens in the Time Domain, Phys. Rev. Lett. 109, 097405 (2012).

[50] M. Xiao, X. Huang, H. Liu and C.T. Chan, Enhancement of polarizabilities of cylinders with cylinder-slab resonances, Scientific Reports 5, 8189 (2015). 